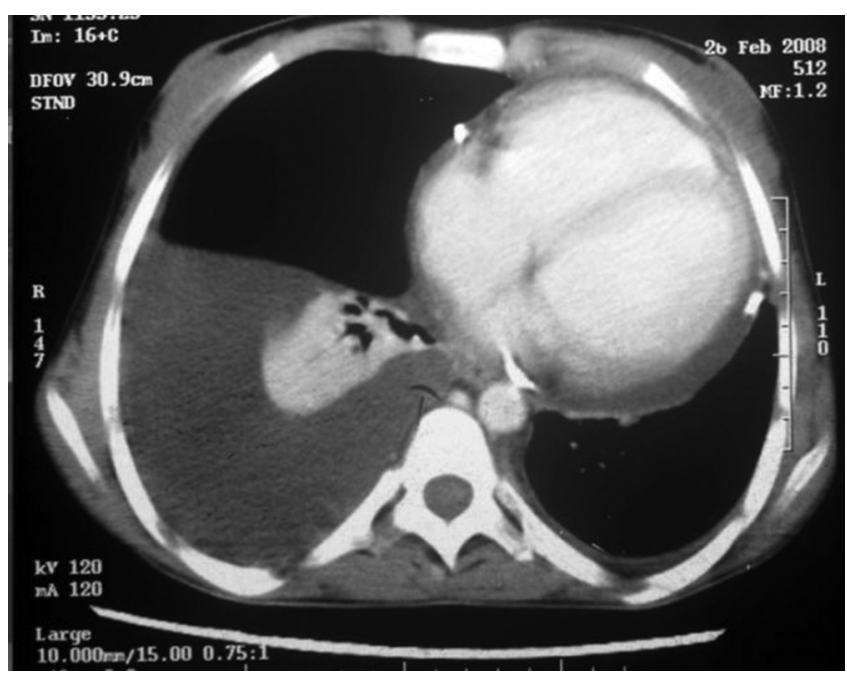

Abstract 133 Figure 1 Pericardial effusion and pleural effusion

hepatomegaly and ascites.She was initiated on decongestive measures with furosemide and digoxin.Chest X-ray showed cardiomegaly(cardiothoracic ratio 68\%);2D-echocardiography (2D-ECHO) revealed cardiomegaly,pericardial effusion and features consistent with cardiac tamponade.She underwent emergency pericardiocentesis, $400 \mathrm{ml}$ of serosanguineous fluid was drained followed by prompt clinical improvement.Investigations showed hemoglobin $90 \mathrm{~g} / \mathrm{L}$; total leukocyte count 1.3 $\times 106 / \mathrm{L}$ and lymphocyte count $0.18 \times 106 / \mathrm{L}$;platelets of $356 \times 109 /$ L.Urinalysis and renal function tests were unremarkable.Tuberculin skin test was non-reactive.Thyroid function tests were normal.In view of febrile illness, malar rash, photosensitivity, pericardial effusion,cardiac tamponade and lymphopenia,SLE was considered. Work-up showed positive antinuclear antibody (ANA)4+diffuse ANA positivity;positive anti-double stranded DNA(anti-dsDNA)-60(normal:4.2 IU/ $\mathrm{ml}$ );C3 $51.8 \mathrm{mg} \%$ (normal $50-150 \mathrm{mg} \%$ ).Skin biopsy revealed IgG and C3 deposits.Pericardial fluid analysis revealed low complement(C3<12 mg\%);ANA positivity and positive LE cells.Bacterial culture of pericardial fluid and polymerase chain reaction to Mycobacterium tuberculosis were negative.Pericardial fluid showed no malignant cells on cytological examination.A diagnosis of SLE was offered based on positive clinical and immunologic findings.Pulse intravenous methylprednisolone was given $(30 \mathrm{mg} / \mathrm{kg} /$ day for 5 days $)$ followed by oral prednisolone(starting at $2 \mathrm{mg} / \mathrm{kg} /$ day and tapered thereof).2D-ECHO showed a reduced volume of pericardial fluid with no additional reaccumulation and normal heart function. She remained well for the next 3 years on hydroxychloroquine. However,at age of 17 years she presented with respiratory distress,oral ulcerations, right pleural effusion and recurrence of pericardial effusion and cardiac tamponade.This required pigtail insertion for pericardiocentesis for 8 days.There was laboratory evidence for disease activity(antids-DNA index 2.24;normal <1.1).She responded to oral prednisolone $(2 \mathrm{mg} / \mathrm{kg} / \mathrm{day})$.A similar episode occurred a year later at 18 years with pericardial and pleural effusion and for this she was given intravenous methylprednisolone pulses for 5 days.In view of recurrent pericarditis, she has been initiated on colchicine $(1 \mathrm{mg} /$ day $)$ following which there has been no recurrence of symptoms.

Conclusions Pericardial effusion with cardiac tamponade at presentation of childhood lupus is distinctly unusual and so is the rarity of recurrent pericardial effusion with cardiac tamponade.Our patient continued to have recurrences on follow-up that responded to glucocorticoids.

Funding Source(s): None

\section{MORTALITY RISK PREDICTION IN LUPUS PATIENTS COMPLICATING WITH INVASIVE INFECTION IN THE EMERGENCY DEPARTMENT}

${ }^{1} Y_{i}$ Chen*, ${ }^{2}$ Wanlong Wu, ${ }^{2}$ Shuang Ye, ${ }^{3}$ Fangfang Sun, ${ }^{1} J u n$ Ma. ${ }^{1}$ Department of Emergency, South Campus, Ren Ji Hospital, School of Medicine, Shanghai Jiao Tong University; '2Department of Rheumatology, South Campus, Ren Ji Hospital, School of Medicine, Shanghai Jiao Tong University; ${ }^{3}$ Ren Ji Hospital, South Campus, Shanghai Jiaotong University

\subsection{6/lupus-2019-Ism.134}

Background Infection remains an important and leading cause of mortality in lupus. We aimed to establish a clinical prediction model for the 3 month all-cause mortality of patients with systemic lupus erythematosus (SLE) and invasive infection in the emergency department.

Methods SLE patients classified by 1997 ACR criteria complicating with invasive infection when admitted into the emergency department of our center between May 2015 and June 2018 were included. Invasive infection was defined as deep infection with definite evidence of pathogens or globally judged by the treating physician combining symptoms, lab and imaging tests. Patients clinical and laboratory characteristics at admission were retrospectively collected as baseline data. The outcome was all-cause death within 3 months since baseline.

Candidate predictors for multivariable logistic regression were selected by expert opinion based on clinical significance, previous studies and feasibility. A prediction model for allcause mortality was established by combining independent predictors and evaluated by Receiver Operating Characteristic (ROC) curve analysis.

Results A total of 50/130 (38.5\%) included patients died cumulatively within 3 month follow-up. Patients were predominantly female (91\%) with a mean disease duration of 6.6 years. One hundred and eight (83.1\%) patients had lung infection, while $23(17.7 \%)$ patients had blood stream infection.

In the final multivariable logistic regression model, lymphocyte count $<800 / \mathrm{ul}$, urea $>7.6 \mathrm{mmol} / \mathrm{L}$, maximum prednisone dose in the past60 mg/d, qSOFA score and age at admission were independently predictive for all-cause mortality. However, the history of hydroxychloroquine use was protective.

In a combined prediction model, the six predictors were weighted by OR values, making the LUPHAS score ranging from 5 to 22 (table). All patients could be categorized to three group: low-risk (score 5-10), medium-risk (score 11-16) and high-risk (score 17-22). The mortalities were $11.1 \%$ (4/ $36), 32.8 \%(22 / 67)$ and $100 \%(22 / 22)$ in low-risk, mediumrisk and high-risk patients, respectively. ROC curve analysis indicated that LUPHAS score could effectively predict all-cause mortality in this population $(\mathrm{AUC}=0.8595 \% \mathrm{CI}=0.78-0.92$ ). Furthermore, LUPHAS score performed better than the sole qSOFA score $(\mathrm{AUC}=0.6995 \% \mathrm{CI}=0.59-0.78)$ in our cohort. The discriminatory performance of LUPHAS score was also superior than CURB-65 score (AUC $=0.6995 \% \mathrm{CI}=0.59-0.80$ ) in the subgroup of patients with lung infection $(n=108)$.

Conclusions In this large emergency cohort of lupus patients complicating with invasive infection, an impressive high 
Abstract 134 Table 1 Establishment of the LUPHAS scoring system

\begin{tabular}{|c|c|c|}
\hline & Predictors & Points \\
\hline \multirow[t]{3}{*}{$\mathbf{L}$} & Lymphocyte count & \\
\hline & $\geq 800 / \mathrm{ul}$ & 1 \\
\hline & $<800 /$ ul & 4 \\
\hline \multirow[t]{3}{*}{ u } & Urea & \\
\hline & $\leq 7.6 \mathrm{mmol} / \mathrm{L}$ & 1 \\
\hline & $>7.6 \mathrm{mmol} / \mathrm{L}$ & 4 \\
\hline \multirow[t]{3}{*}{$\mathbf{P}$} & Maximum Prednisone dose in the past & \\
\hline & $<60 \mathrm{mg} / \mathrm{d}$ & 1 \\
\hline & $\geq 60 \mathrm{mg} / \mathrm{d}$ & 5 \\
\hline \multirow[t]{3}{*}{ H } & History of Hydroxychloroquine use & \\
\hline & Yes & -1 \\
\hline & No & 0 \\
\hline \multirow[t]{5}{*}{ A } & Age, $y$ & \\
\hline & $\leq 20$ & 1 \\
\hline & $21-40$ & 2 \\
\hline & $41-60$ & 3 \\
\hline & $>60$ & 4 \\
\hline \multirow[t]{4}{*}{$\mathbf{S}$} & qSOFA score & \\
\hline & 0 & 0 \\
\hline & 1 & 3 \\
\hline & $\geq 2$ & 6 \\
\hline
\end{tabular}

mortality was recorded, underlining that special attention should be paid to these patients. Several independent predictors for all-cause mortality were successfully identified in our study. The real-world evidence-based LUPHAS score might be a promising tool for risk stratification in clinical practice.

Funding Source(s): None

\section{INFLUENCE OF GENETIC VARIANTS ON AVASCULAR NECROSIS IN PATIENTS WITH SYSTEMIC LUPUS ERYTHEMATOSUS}

${ }^{1}$ So-Young Bang*, ${ }^{2}$ Youngho Park, ${ }^{1}$ Hyuk-Hee Kwon, ${ }^{3}$ Kwangwoo Kim, ${ }^{1}$ Hye-Soon Lee, 'Sang-Cheol Bae. 'Department of Rheumatology, Hanyang University Hospital for Rheumatic Diseases; ${ }^{2}$ Clinical Research Center for Rheumatoid Arthritis; ${ }^{3}$ Department of Biology, Kyung Hee University, Republic of Korea

10.1136/lupus-2019-Ism. 135

Background Avascular necrosis (AVN) incompletely understood genetic architecture in patients with systemic lupus erythematosus (SLE). The aims of this study were to investigate genetic risk factors, biological and functional pathways associated with the risk of AVN in a large SLE cohort in Korea.

Methods Patients with SLE were enrolled in the Hanyang BAE Lupus cohort, in whom damage was measured annually according to the Systemic Lupus International Collaborating Clinics/American College of Rheumatology Damage Index (SDI). We conducted an association study to determine genetic risk factors for AVN in 612 SLE cases that genotyped by high-density immune-loci genotyping array (Immunochip) and genome-wide association study (GWAS) using Illumina HumanOmni1-Quad array.

Results Symptomatic AVN was the most common type of musculoskeletal damage $(14.2 \%, \mathrm{n}=87)$ among 612 SLE patients in Korea. In analysis of clinical factors, use of a high cumulative corticosteroid dose (OR 3.62, $\mathrm{p}=0.015$ ) significantly increased the risk of $\mathrm{AVN}$ in multivariable analysis. We found the top 10 single-nucleotide polymorphisms (SNPs) associated with AVN $(p<5.0 \times 106)$ in SLE patients with AVN after 